\title{
Erratum to: A survey on offline scheduling with rejection
}

\author{
Dvir Shabtay • Nufar Gaspar • Moshe Kaspi
}

Published online: 23 January 2015

(C) Springer Science+Business Media New York 2015

\section{Erratum to: J Sched (2013) 16:3-28 \\ DOI 10.1007/s10951-012-0303-z}

Below, we list four oversights that were found in our paper.

1. The result appearing in Theorem 8 (page 10) was presented earlier (in a slightly different form) by Koulamas (2011). We overlooked this result, and want to give him the proper credit for it.

2. On page 4, we include the following statement: "if $F_{1}$ is indeed the dominant criterion then using the lexicographical approach will yield a non-balanced solution for which $A=\emptyset$ and $\bar{A}=J$." However, this is not always the case. Consider, for example, an instance of the $1|r e j| \epsilon\left(F_{1}, R C\right)$ problem with $F_{1} \in\left\{T_{\max }, \Sigma T_{j}, \Sigma U_{j}\right\}$, where it is possible to schedule the entire set of jobs $(J)$ such that all jobs are non-tardy, i.e., such that $T_{\max }=0$. For such an instance, using the lexicographical approach with $F_{1}$ being the dominant criterion will yield a solution in which $A=J$ and $\bar{A}=\emptyset$, thereby contradicting our statement.

3. The value of the positional penalty for $\delta_{1} \sum \sum\left|W_{i}-W_{j}\right|$ $+\delta_{2} \sum W_{j}$ in Table 4 (page 14) should be replaced by $\xi_{j}(k)=\delta_{1}(j-1)(k-j+1)+\delta_{2}(j-1)$.

The online version of the original article can be found under doi:10.1007/s10951-012-0303-z.

D. Shabtay $(\bowtie) \cdot$ N. Gaspar · M. Kaspi

Department of Industrial Engineering and Management, Ben-Gurion University of the Negev, Beer-Sheva, Israel

e-mail: dvirs@bgu.ac.il

N. Gaspar

e-mail: nufarg@bgu.ac.il

M. Kaspi

e-mail: moshe@bgu.ac.il
4. On page 16, we mention that Steiner and Zhang (2011) present a pseudo-polynomial time optimization algorithm to solve the $1|r e j| \sum_{J_{j} \in A} T_{j}+R C$ problem in $O\left(n^{4}\left(\sum_{j=1}^{n} p_{j}\right)^{3}\right)$ time, which further implies that this algorithm can serve as an $O\left(n^{7}\right)$ time algorithm to solve the special case of equal processing times. However, we failed to observe that Steiner and Zhang provided a faster $O\left(n^{2}\right)$ time optimization algorithm to solve this special case.

Acknowledgments The authors want to thank Prof. Christos Koulamas for pointing out the above errors.

\section{References}

Koulamas, C. (2011). A unified solution approach for the due date assignment problem with tardy jobs. International Journal of Production Economics, 132, 292-295.

Steiner, G., \& Zhang, R. (2011). Revised delivery-time quotation in scheduling with tardiness penalties. Operations Research, 59, 1504-1511. 